\title{
EFFECT OF LASER THERAPY IN INFLAMED TISSUE BY MEDICATIONS BASED ON IODOFORM LASER THERAPY IN INFLAMED TISSUE
}

\author{
${ }^{1}$ Pablicia Juliana Santos Tomaz, ${ }^{1}$ Michelle de Paula Farias, ${ }^{1}$ Marta Rabello Piva, \\ ${ }^{2}$ Ricardo Luiz Cavalcanti de Albuquerque Junior and ${ }^{1}$ Maria Amalia Gonzaga Ribeiro \\ ${ }^{1}$ Department of Dentistry, Faculty of Dentistry, Federal University of Sergipe, Aracaju, Brazil \\ ${ }^{2}$ Department of Dentistry, Faculty of Dentistry, Tiradentes University, Aracaju, Brazil
}

Received 2012-09-25, Revised 2013-01-15; Accepted 2013-03-08

\begin{abstract}
This study aimed to evaluate the inflammatory response of the subcutaneous tissue of twenty rats when the use of iodoform with different drugs or not associated with laser photobiomodulation. In the dorsum of rats were introduced into tubes containing: Iodoform and calcium hydroxide $\left(\mathrm{Ca}(\mathrm{OH})_{2}\right)$; Iodoform and $\mathrm{Ca}(\mathrm{OH})_{2}$, associated with Laser; Iodoform and Otosporin; Iodoform and Otosporin, associated with Laser. The animals were euthanized eight and fifteen days after surgery, the parts were removed and processed in the laboratory with staining with Hematoxylin-Eosin (HE) and Picrosirius Red. The statistical significances of the inflammatory infiltrate and quantification of collagen fibers were measured by ANOVA-Tukey test. The association between $\mathrm{Ca}(\mathrm{OH})_{2}$ and iodoform with determined the best response in the host tissues. The low level laser therapy was effective in modulating the inflammatory response and deposit collagen fibers.
\end{abstract}

Keywords: Inflammation, Iodoform, Lasers Semiconductors, Wistar Rats

\section{INTRODUCTION}

The main concern of Endodontics is to promote the complete cleaning and sanitation of the root canal system, aiming the success of endodontic therapy, because the presence of microorganisms is a key factor for installation and perpetuation of pulp and periapical main pathologies. The use of intracanal medication between sessions aims to enhance the process of sanitation achieved during the preparation of the canal (Karim et al., 2007).

In the hopes of supplying the limitations of intracanal medication, associations of different medications are praised, with the objective of meeting the different requirements of pulp-periapical conditions (Lana et al., 2009).

Among the various medications that are used on endodontic therapy, the iodoform has excellent antimicrobial activity that decreases purulent secretions, which may be important in the case of exsudate present in the periapical region, being easily removed when extravasated to the periapex (Mortazavi and Mesbahi, 2004).

In endodontic practice the association of corticosteroidantibiotic can also be highlighted as an intracanal medication, being the Otosporin ${ }^{\circledR}$ the most widely used, for its antiinflamatory activity (Silva et al., 2004).

Other medicament widely used as a intracanal medication in the endodontic practice is $\mathrm{Ca}(\mathrm{OH})_{2}$, which has long been subject of study due to its antimicrobial properties, solvent of organic matter and inducer of formation of mineralized tissue (Garcia et al., 2008).

With the scientific-technical advance, different devices were introduced in the dental practice, among them stands out the Laser. Studies have shown that the laser photobiomodulation positively contributes in various biological activities by interacting with the tissue, such as: Increasing local microcirculation, reducting the number of inflammatory cells, analgesic effects, stimulating proliferation of epithelial cells and

Corresponding Author: Pablícia Juliana Santos Tomaz, Department of Dentistry, Faculty of Dentistry, Federal University of Sergipe, Aracaju, Brazil 
fibroblasts, as well the increasing collagen synthesis (Dantas et al., 2011; Rodrigues et al., 2009).

At cellular level, the low power laser causes biochemical, bioelectric and bioenergetic changes, works on increasing metabolism, cell proliferation and maturation, on the amount of granulation tissue and in the reduction of inflammatory mediators, thus speeding up the healing process. The molecular absorption of laser light allows an increase in cellular metabolism, characterized by stimulation of photoreceptors in the mitochondrial respiratory chain, changes in levels of cellular ATP, release of growth factors and collagen synthesis (Silva et al., 2007).

Thereby, the objective of this study was to evaluate morphological and morphometrically the reaction in the subcutaneous tissues of mice, amid the use of iodoform associated with different medication and subjected to Laser photobiomodulation.

\section{MATERIALS AND METHODS}

\subsection{Ethical Aspects}

According to the guidelines described in the "Guide for the Care and Use of Laboratory Animals" it is sure that all animals received humane care throughout all stages of experimentation. In addition, the study protocols were approved by the local National Research Council before the beginning of the experiments (registration \#170211).

\subsection{Preparation of the Tubes}

Polyethylene tubes (urethral catheter with an internal diameter of four $\mathrm{mm}$ ) were sectioned at intervals of eight $\mathrm{mm}$ (Garcia et al., 2008). It must be pointed out that one of the extremities was sealed with cyanoacrylate esther gel (Super Bonder®, Aachen, Germany) in order to prevent the test paste came in contact with the subcutaneous tissue of the mouse, making it, so, the side control experiment. The tubes obtained were autoclaved in metal canisters at a temperature of $120^{\circ} \mathrm{C}$ for $20 \mathrm{~min}$.

\subsection{Surgical Procedure}

Twenty male mice (Rattus norvegicus, Wistar lineage) weighing 250-300g were used in this study. The animals were intraperitonially anesthetized with a solution of Xylazine-Ketamine Hydrochloride (100-5 mg $\mathrm{kg}^{-1}$ ), later trichotomized on the dorsal region. The antisepsis of the surgical field was then performed with the use of polvidine-iodine $10 \%$ topical solution (LM FARMA®). Four incisions were made at five $\mathrm{mm}$ wide, two near the lower back and the other two near the scapular region, for insertion of the medicaments.

\subsection{Preparation of Pastes (Medication Associations)}

The medicaments Paste A (3.0 g of iodoform associated with $1.75 \mathrm{~mL}$ of Otosporin $\left.{ }^{\circledR}\right)$ and Paste B (3.0 g iodoform associated with $3.0 \mathrm{~g}$ of $\mathrm{Ca}(\mathrm{OH})_{2}$ and $1.75 \mathrm{ml}$ of serum) were manipulated into a sterile glass plate. Polyethylene tubes were filled with the help of a sterile file (\#25 Dentsply-Maillefer, RJ, Brazil) immediately after manipulation of the medication. Then the tubes were inserted and adapted into the surgical cavity with the side containing the paste towards the animal's head and the control side sealed with methyl cyanoacrylate (Super Bond®, Aachen, Alemanha) towards the caudal region. After placing the tubes containing different medication, the continuous suture of the incision was performed with nylon 3-0 monofilament thread in the skin and then performed new antisepsis with iodine alcohol in the operated area (Sassioto et al., 2004). The mice were randomly distributed into four groups according to medication used and, the association or not with laser photobiomodulation, namely: $\mathrm{IH}(\mathrm{n}=5)$ tube containing iodoform, serum and $\mathrm{Ca}(\mathrm{OH})_{2}$; IHL $(\mathrm{n}=$ 5) tube containing iodoform, serum and $\mathrm{Ca}(\mathrm{OH})_{2}$, associated with the laser photobiomodulation; IO $(\mathrm{n}=5)$ tube containing iodoform and Otosporin $\AA$; IOL $(n=5)$ tube containing iodoform and Otosporin ${ }^{\circledR}$ associated with the laser photobiomodulation. It should be emphasized that the side of the polyethylene tube sealed with Super Bond® opposite to test medication was used as Control (CTR) and this, when irradiated, was called LTP.

\subsection{Low Level Laser Therapy (LLLT) Protocol}

According to the parameters of Diode Laser irradiation (Twin Laser-InGaAlP/MMOPTICS, São Carlos, São Paulo, Brazil), the wavelength used is included in the red spectrum, $\chi 660 \mathrm{~nm}$. The Laser light radiated at four points in each surgical wound made, being one caudal, another radial and two lateraltranscutaneous, by contact and perpendicular to the long axis of the back of the animal. The groups in which laser treatment was applied received a total of four irradiations with $48 \mathrm{~h}$ interval between sessions, being the first immediately after surgery, according to the protocol described in Table 1 (Ribeiro et al., 2009a).

\subsection{Euthanasia of the Animals and Preparation of Samples}

The animals were euthanized in the eighth and fifteenth days, by an overdose of Thio-Pental (Barbiturate-CRYSTALIA ®) $0.43 \mathrm{~mL} \mathrm{~kg}^{-1}$. 
Table 1. Device and parameters of laser irradiation

\begin{tabular}{lll}
\hline Irradiation parameters & IOL Group & IHL Group \\
\hline Emission mode & Continous-CW & Continous-CW \\
Wavelength & $660 \mathrm{~nm}$ & $660 \mathrm{~nm}$ \\
Medium active & InGaAlP & InGaAlP \\
Optical power of the Laser (output) & $40 \mathrm{~mW}$ & $40 \mathrm{~mW}$ \\
Area of the beam spot & $4 \mathrm{~mm}$ & $4 \mathrm{~mm}$ \\
Area $\left(\mathrm{cm}^{2-} \mathrm{A}=\pi \cdot \mathrm{r}^{2}\right)$ & $0,04 \mathrm{~cm}^{2}$ & $0,04 \mathrm{~cm}^{2}$ \\
Power Density & $1 \mathrm{~W} / \mathrm{cm}^{2}$ & $1 \mathrm{~W} / \mathrm{cm}^{2}$ \\
Energy Density & $20 \mathrm{~J} / \mathrm{cm}^{2}$ & $20 \mathrm{~J} / \mathrm{cm}^{2}$ \\
Time per point & $20 \mathrm{sec}$ & $20 \mathrm{sec}$ \\
Total energy & $4 \mathrm{~J}$ & $4 \mathrm{~J}$ \\
Beam divergence perpendicular to the junction & $17^{\circ}$ & $17^{\circ}$ \\
Tip angle & $50^{\circ}$ & $50^{\circ}$ \\
\hline
\end{tabular}

Source: UFS/2011

The parts removed were subjected to routine laboratory for $\mathrm{HE}$ and specific Picrosirius Red stainings, for coloring the collagen fibers.

\subsection{Inflammatory Response Evaluation}

The histological cuts stained with HE were used for descriptive analysis of the profile of the inflammatory infiltrate. The inflammatory response was evaluated this way: +1 (when inflammatory cells represent less than $10 \%$ of the cell population found in the surgical wound); +2 (when inflammatory cells represent between $10-50 \%$ of the cell population observed in the area of surgical wound); +3 (when inflammatory cells represent more than $50 \%$ of the cell population observed in the area of the surgical wound). With this, we classify as +1 mild inflammatory infiltrate, +2 moderate inflammatory infiltrate, +3 intense inflammatory infiltrate.

\subsection{Morphological Description of the Collagen Fibers}

The histological cuts stained with Pricosiris Red and analyzed under polarized light were used for qualitative analysis of the collagen fibers deposition (Dantas et al., 2011; Carneiro et al., 2005). The collagen fibers were analyzed according to bi-refraction pattern phenomenon for characterization of the type of collagen fiber (greenish/greenish yellow-fiber type-III or orangish/reddish-orange - type I fibers), the morphological appearance (short or long, thin or thick) and arrangement of collagen fibers (reticular, parallel or interlaced).

\subsection{Quantitative Analysis of Collagenization}

The area determined for quantitative analysis of collagen fiber deposition, in the region around the implanted tubes, was determined in randomly selected fields and the quantification performed by an image analysis system. The system used consists of a CCD Sony DXC-101 camera, coupled to an Olympus CX31 microscope. Through scanning systems (Olympus C7070 WideZoom), the images were transferred to a computer (Pentium $133 \mathrm{MHz}$ ) and processed in a software (UTHSCSA Image Tool Version 3.0). A total of ten fields for each group, magnified at $100 \times$, were analyzed. The thresholds for the collagen fibers were established for each image and the area occupied by the fibers was determined by digital densitometric, setting the measuring threshold level up to the color density different from the collagen fibers. The area occupied by the fibers was divided by the total area of the field. The results were expressed as percentage of skin area fraction occupied by collagen fibers.

\subsection{Statistical Analysis}

The statistical significances of the inflammatory infiltrate and quantification of collagen fibers were measured by analysis of variance (one-way ANOVA) and if there was a difference of variance, it was applied the Tukey Test. The values in which $\mathrm{p} \leq 0.05$ were considered significant.

\section{RESULTS}

In all studied groups, it was observed the presence of inflammatory reaction of variable intensity, predominantly chronic, in the area surrounding the opening of the placed tubes. In some specimens, foreign body giant cells were identified, suggesting the formation of small granulomas in all analyzed groups. In addition, some specimens where tubes containing iodoform and $\mathrm{Ca}(\mathrm{OH})_{2}$ association were implanted, it was possible to observe necrotic areas surrounding the opening of the tubes containing the medications, as well as sparse dystrophic calcification foci (Fig. 1).

As shown in Fig. 2, it was observed in eight days, induction of inflammatory reaction significantly more intense in the IO and $\mathrm{IH}$ groups compared to the CTR group ( $\mathrm{p}=0.002$ and 0.001 respectively). 

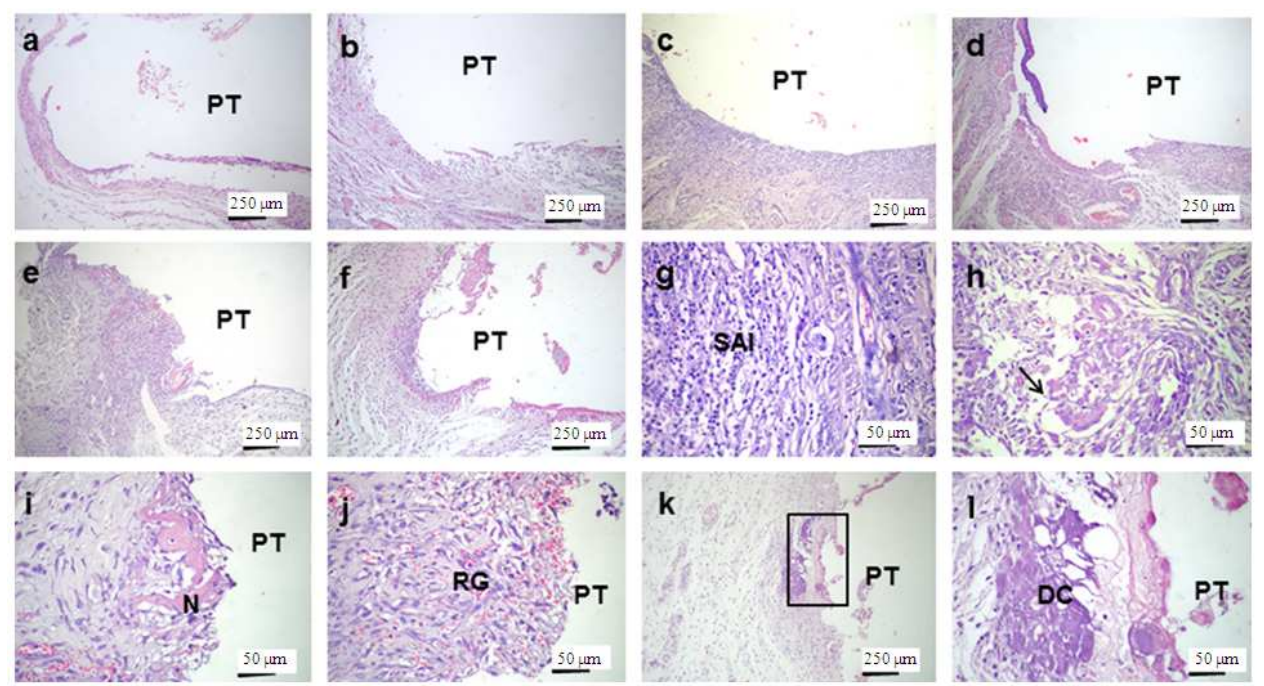

Fig. 1. Photomicrograph in HE showing the Polyethylene Tubes (PT) implanted. Observe the inflammatory reaction surrounding the openings of the tubes (a) CTR, (b) LTP, (c) IO, (d) IOL, (e) IH and (f) IHL. (g) Detail exuberant Subacute Inflammation $(\mathrm{SAI})$ in IO and $(\mathrm{h})$ granuloma rich in giant cells (arrows) in IOL. (i) Area of necrosis $(\mathrm{N})$ and reaction (j) grain well developed in IH. (k) Highlight focus of dystrophic calcification in IHL. (1) Detail of previous figure, showing irregular areas of Dystrophic Calcification (DC)
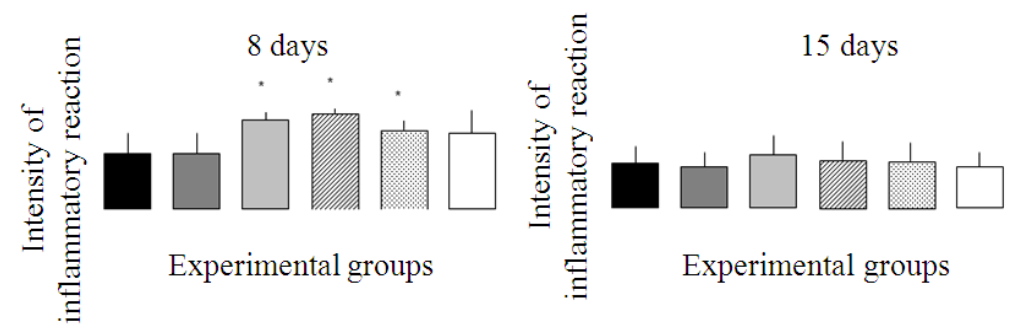

Fig. 2. Histogram representing the intensity of inflammation categorized groups in scores in eight days after implantation of the tubes. (*)Statistically significant difference with the group CTR and LTP

In the groups treated with LLLT, it was evidenced that, although in IOL the inflammatory reaction was significantly more intense than in LTP $(p=0.03)$, in the group IHL, the intensity of this reaction was statistically similar to the LTP and CTR groups $(\mathrm{p}=0.07)$. In 15 days, the intensity of inflammatory reaction varied from mild to moderate in all groups studied. It was not evidenced difference between them $(\mathrm{p}=0.588)$.

Regarding the pattern of collagen fiber deposition, on the eighth day, it was observed in the CTR, LTP, IO, IOL and IH groups predominance of type III collagen fibers, short and slender, slightly condensed and arranged predominantly interlaced. However the IHL group had a higher formation of type I fibers, also short and a bit thicker than the other groups, slightly condensed and arranged predominantly interlaced (Fig. 3.1). On the fifteenth day, it was observed in all groups the presence of collagen fibers I, short but thicker in appearance when compared to the eight days ones, arranged interlaced and showing slight condensation (Fig. 3.2).

As for the quantitative analysis of deposited collagen fibers no significant difference between groups nor in eight $(\mathrm{p}=0.50)$ nor in 15 days $(\mathrm{p}=$ 0.68 ) about the iodoform and $\mathrm{Ca}(\mathrm{OH})_{2}$ association, combined or not with LLLT. However, about the iodoform and Otosporin ${ }^{\circledR}$ association, although no differences have been observed between the groups, irradiated or not, during eight days $(\mathrm{p}=0.24)$ in 15 days, the IOL group had a higher fibroplasia compared to non-irradiated groups IO ( $\mathrm{p}=$ $0.01)$ and CTR ( $\mathrm{p}=0,002)$ and collagen deposition similar to the LTP group $(\mathrm{p}=0.57)$. 

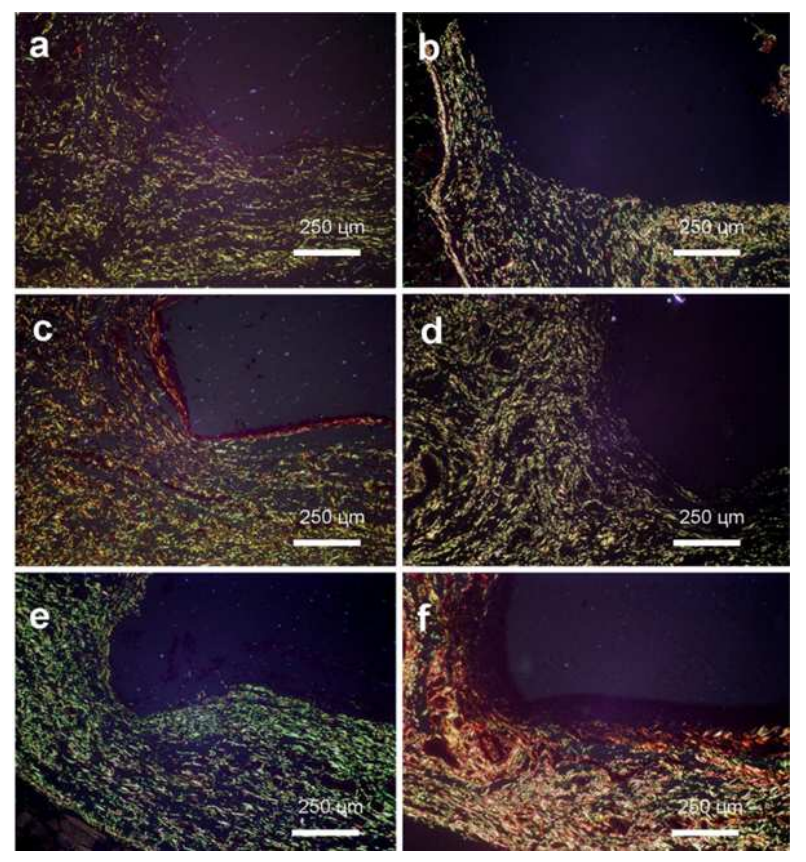

Fig. 3.1. (a) CTR and (b) LTP predominance of type III fibers; (c) IO and (f) IHL, predominance of type I fibers, (d) and (e) only type III
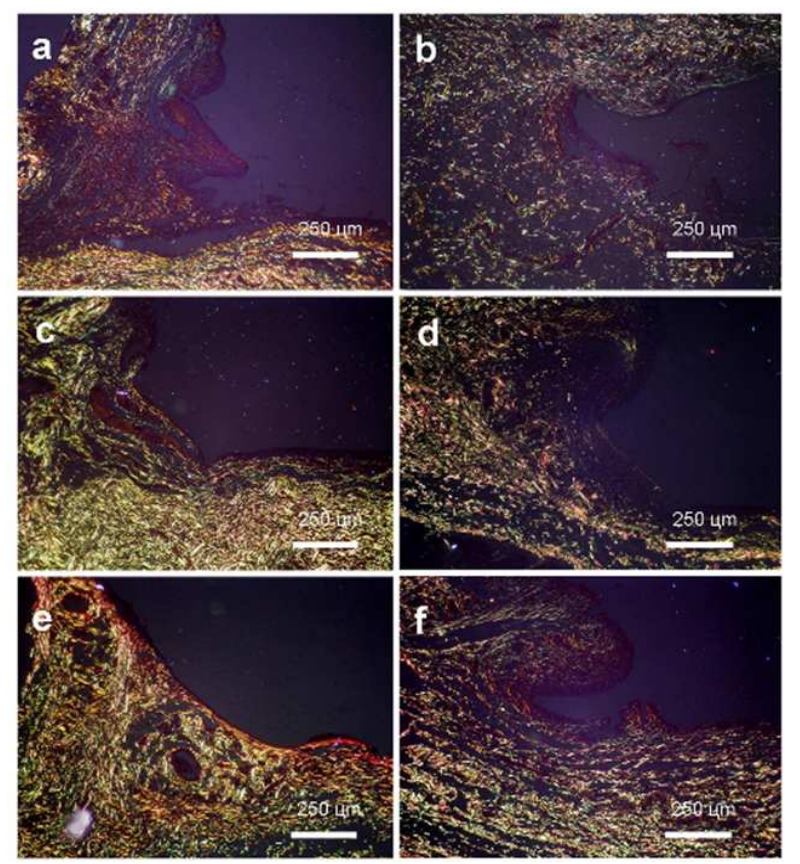

Fig. 3.2. (a) CTR only type I collagen; (b) and (c) predominance of type III fibers; (d), (e) and (f) predominance of type I fibers

\section{DISCUSSION}

The implementation methodology of polyethylene tubes in the connective tissue of mice used in this study was based on Torneck (1966) experiments, which demonstrated the acceptability of this material by the subcutaneous connective tissue of animals, as well as, since it is a controlled method with few variables and low cost (Garcia et al., 2008; Valera et al., 2004). Besides, polyethylene tubes mimic the conditions of the root canal, since only the final portion of the tube, corresponding to the root apex, the test material will contact the tissue and the opposite side sealed with biocompatible material will serve as control (Zmener et al., 1988). Another fact that must be highlighted refers to the experimental model used in this research, because the rapid metabolism of these animals makes it possible to obtain results in a short period of time and also it has similarity to the human skin (Garcia et al., 2008).

The choice of two times sacrifice of animals (eight and fifteen days) were based on biological phenomena which characterize the inflammation process and repair in these periods (Ribeiro et al., 2009b).

Among the medications used in Endodontics, several stand out for their biological properties, although the professional does not have yet in their arsenal a medicament which performs bactericidal effective and has low cytotoxicity, making associations be performed on an ambulatory basis.

During the association of the medications iodoform and $\mathrm{Ca}(\mathrm{OH})_{2}$, it was used an aqueous and inert vehicle, the serum, producing circumstances so that the inherent properties of the tested medications were able to perform their activities without any influence from chemically active vehicles. However, in the association of iodoform to Otosporin ${ }^{\circledR}$, this one was used as biologically active vehicle due to inflammatory intensity modulation properties and discrete antimicrobial activity. However, it is worth to point out that associations between medications are not always beneficial and, depending on the association, it can promote the enhancement or even the suppression one of the medications used.

About the association of $\mathrm{Ca}(\mathrm{OH})_{2}$ with iodoform, irradiated or not, in at the different times analyzed, it was observed in some specimens, areas of necrosis and dystrophic calcification. This fact seems to be related to the direct action of $\mathrm{Ca}(\mathrm{OH})_{2}$ with the tissue, causing hence, physical-chemical alterations of the extracellular matrix, cleavage of glycoproteins and protein denaturation, forming areas of necrosis and on these, there 
is a rapid precipitation of crystals, depositing calcium and phosphorus ions, thereby inducing the formation of dystrophic calcification (Queiroz et al., 2005).

In $\mathrm{IO}$ and $\mathrm{IH}$ groups, in eight days, there was a significant increase in the intensity of inflammatory reaction when compared to the CTR group, suggesting that, despite exhibiting satisfactory properties for endodontic use, $\mathrm{Ca}(\mathrm{OH})_{2}$ and iodoform have irritant properties, the first by the presence of iodine in its composition and the second by promoting high alkalisation.

In this study, the association of iodoform with Otosporin ${ }^{\circledR}$ did not seem to influence the magnitude of the inflammatory response. Corroborating these findings, Silva et al. (2004), in this comparative study on the evaluation of anti-inflammatory activity of natural and endodontic medications, among them Otosporin ${ }^{\circledR}$, concluded that the activity of this over the intensity of inflammatory reaction did not show satisfactory efficiency. This finding could be related to the fact that the anti-inflammatory activity of Otosporin ${ }^{\circledR}$ would be limited to early acute phases of inflammation had little influence on the later stages of the inflammatory response. Furthermore, Goodwin et al. (1986) demonstrated that the immunosuppressive action of glucocorticoids can be reversed by a significant increase in tissue levels of leukotrienes, especially LTB4. Thus, the irritant action of iodoform could have induced a more severe inflammatory response and consequent increase in synthesis and release of LTB4, minimizing the antiinflammatory effects of Otosporin ${ }$.

The LLLT significantly promoted the reduction of the intensity of inflammatory reaction in the IHL group. It is attributed this decrease of the intensity of the inflammatory reaction of the irradiated groups to the properties of biological mechanisms and stimulation of LLLT, which participate in the cicatricial repair stages in damaged tissues, including inducing the release of cytokines and growth factors, stroma cell proliferation and inhibition of Cyclooxygenase synthesis (COX-2) and the proinflammatory cytokines, modulating the inflammatory response, thereby accelerating the repair process (Ribeiro et al., 2009a).

Paradoxically, the combination of LLLT and medication contents corticosteroids (IOL group) did not significantly reduce the magnitude of the inflammatory reaction. The explanation for this phenomenon seems to lie in the biomodulatory activity of LLLT in the early stages of inflammation. Pereira et al. (2002) demonstrated that the laser in the red spectrum promotes a significant increase in mast cells degranulation in the first $24 \mathrm{~h}$ after tissue injury, generating a transitory amplification of the acute inflammatory response, followed by contents the substantial reduction of neutrophil after $36 \mathrm{~h}$. These data suggest that LLLT modulates the inflammatory response in order to accelerate the acute phase and anticipate the chronification of the inflammatory process, an essential phenomenon for the installation of the granulation reaction and start the repair process (Ribeiro et al., $2009 \mathrm{~b}$ ). Thus, the presence of steroids in the association, a chemical compound with important anti-inflammatory effect $^{18}$, could have minimized the modulatory action of LLLT in the early stages of the inflammatory process and, consequently, allowing the acute inflammatory phase to be prolonged for a longer time, which would justify a greater intensity of this pathophysiologic response in eight days in the IOL group compared to the group without steroids (IHL).

The fibrotic response of the tissues to the endodontic medications constitutes an important variable to ensure the success of the endodontic treatment, since the induction of cicatricial fibrosis would prevent the natural course of bone regeneration in cases of periapical lesion. The dynamics of fibroplasia was evaluated by descriptive and quantitative analyzes of the newly formed collagen. Sections stained by Pricosirius Red enabled to evaluate the collagen type in the samples. The type III collagen form thin and delicate fibers, loosely arranged showing a weak birefringence of greenish and yellowish coloration - while type I collagen form fibers of variable thickness, arranged parallel or interlaced, with yellow-orangish and reddish bi-refringence (Dantas et al., 2011; Carneiro et al., 2005). During the formation of the cicatricial process, collagen type III is initially synthesized and deposited and acts as a framework for migration of endothelial cells that will form the granulation reaction, being replaced by type I occurs as it cicatricial tissue maturation (Carneiro et al., 2005; Fleischmajer et al., 1990). This process has relevance for the dermal resistance in cicatricial area, since the increase in the relation between collagen type I and type III is essential for the tensile strength and mechanical stability of connective tissue (Friedman et al., 1993).

The predominance of type III collagen in most studied groups, in the eighth day, is indicative that the process of fibroplasia was still presented in the early stages. Previous studies analyzing the dynamics of fibroplasia in cicatricial dermal repair have also observed similar results (Albuquerque-Junior et al., 2009; Nunes et al., 2011) and it has been suggested that this type of collagen formed primarily acts as a framework 
for the formation of the fundamental reaction to repair the tissue attacked. However, it was observed the substitution of the type III collagen to the type I collagen molecules by the IHL group. Found similar granulation, a stage was described by Dantas et al. (2011) during the repair process in animals subjected to LLLT in the red spectrum, suggesting that laser irradiation promoted acceleration of collagen fibers maturation process.

In fifteen days, all groups presented a mixed pattern of collagen fibers, however, the LTP and the IO groups presented predominance of type III and the others, of type I. The substitution of type III fibers by type I fibers is an absolutely normal phenomenon in the repair process (Nunes et al., 2011). However, excessive formation of type I fibers can easily lead to undesirable formation of hypertrophic scars and keloids. Moreover, moderate content of thinner collagen fibers appears to provide a low probability of developing keloids, a very desired property (Verhaegen et al., 2009).

In the present study, although we have detected a gradual increase throughout the experimental period, only in the IOL group, in fifteen days, showed a significant increase in the synthesis and deposition of type I collagen, which seems to suggest the establishment of a picture of scar fibrosis. This finding seems to be related to the increased longevity of the inflammatory reaction observed in this group in the first stage of the experiment, which would determine a greater tissue destruction and therefore, a greater need for cicatricial collagen deposition (Ribeiro et al., 2009b). Furthermore, the stimulatory action of LLLT on fibroblasts (Rodrigues et al., 2009) act as co-important factor to substantially increase fibroplasia.

The data presented this study suggest that the combination of LLLT and medication association between $\mathrm{Ca}(\mathrm{OH})_{2}$ and iodoform determined the best response in the host tissues, since it reduced the intensity of inflammation induced by the irritating action of drugs and modulated fibroplasia, promoting acceleration of collagen maturation without however promoting morphological signs of fibrosis. Though, considering the findings of this study, certainly new experiments using the same methodology will be performed with a larger period of time, because then they obtained results will help in clinical practice, reducing the number of changes in intracanal medications.

\section{CONCLUSION}

From the results presented in this study it is concluded that in all groups, the used medications combinations presented irritating properties when in contact with soft tissues.
The LLLT proved to be effective in modulating the intensity of inflammation, as well as the deposition of collagen fibers.

The iodoform and $\mathrm{Ca}(\mathrm{OH})_{2}$ association, associated to the LLLT, showed less damage to the subcutaneous tissues of the animals when compared to the iodoform and Otosporin ${ }^{\circledR}$ association.

\section{REFERENCES}

Albuquerque-Junior, R.L.C., A.L.S. Barreto, J.A. Pires, F.P. Reis and S.O. Ribeiro et al., 2009. Effect of bovine type-I collagen-based films containing red propolis on dermal wound healing in rodent model. Int. J. Morphol., 27: 1105-1110.

Carneiro, C.G., L.U. Sennes, P.H.N. Saldiva, D.H. Tsuji and J.A.X. Filho, 2005. Assessment of collagen deposits after implant of fascia lata and fat in the vocal folds of rabbits: Histomorphometric study. Revista Brasileira Otorrinolaringol., 71: 798-802. DOI: 10.1590/S0034-72992005000600018

Dantas, M.D.M., D.R.R. Cavalcante, F.E.N. Araujo, S.R. Barretto and G.T. Aciole et al., 2011. Improvement of dermal burn healing by combining sodium alginate/chitosan-based films and low level laser therapy. J. Photobiol., 105: 51-59. DOI: 10.1016/j.jphotobiol.2011.06.009

Fleischmajer, R., J.S. Perlish, R.E. Burgesson, F. ShaikhBahai and R. Timpl, 1990. Type I and type III interactions during fibrilogenesis. Annals New York Acad. Sci., 580: 161-175. DOI: 10.1111/j.17496632.1990.tb17927.x

Friedman, D.W., C. Boyd, J.W. Mackenzie, P. Norton and R.M. Oslon et al., 1993. Regulation of collagen gene expression in keloids and hypertrophic scars. J. Surgery Res., 55: 214-222. DOI: 10.1006/jsre.1993.1132

Garcia, L.F.R., R.C.C. Lia, R.A. Lopes, D.A. Oliveira and F.C.P. Pires-de-Souza et al., 2008. Morphological and morphometrical analysis of the subcutaneous tissue of rats submitted to the action of calcium hydroxide paste and Ricinus communis oil. Ciencia Odontologica Brasileira, 11: 47-54.

Goodwin, J.S., D. Atluru, S. Sierakowski and E.A. Lianos. 1986. Mechanism of action of glucocorticosteroids Inhibition of $\mathrm{T}$ cell proliferation and interleukin 2 production by hydrocortisone is reversed by leukotriene B4. J. Clin. Investig., 77: 1244-1250. DOI: 10.1172/JCI112427

Karim, I.E., J. Kennedy and D. Hussey, 2007. The antimicrobial effects of root canal irrigation and medication. Oral Surg. Oral Med. Oral Pathol. Oral Radiol. Endod., 103: 560-569. PMID: 17223590 
Lana, P.E.P., M.F.Z. Scelza, L.E. Silva, A.L. MattosGuaraldi and R.H. Junior, 2009. Antimicrobial activity of calcium hydroxide pastes on Enterococcus faecalis cultivated in root canal systems. Brazilian Dental J., 20: 32-36. DOI: 10.1590/S0103-64402009000100005

Mortazavi, M. and M. Mesbahi, 2004.Comparison of zinc oxide and eugenol and Vitapex for root canal treatment of necrotic primary teeth. Int. J. Paediatric Dentistry, 14: 417-424. DOI: 10.1111/j.1365263X.2004.00544.x

Nunes, P.S., R.L.C. Albuquerque-Junior, D.R.R. Cavalcante , M.D.M. Dantas and J.C. Cardoso et al., 2011. Collagen-based films containing liposomeloaded usnic acid as dressing for dermal burn healing. J. Biomed. Biotechnol., 1-9. DOI: 10.1155/2011/761593

Pereira, N.A., C.P. Eduardo, E. Matson and M.M. Marques, 2002. Effects of low-power laser irradiation on cell growth and procollagen synthesis of cultered fibroblasts. Lasers Surgery Med., 31: 263-267. DOI: 10.1002/1sm.10107

Queiroz, A.M., S. Assed and M.R. Leonardo, 2005. MTA and calcium hydroxide for pulp capping. J. Applied Oral Sci., 3: 126-130. DOI: 10.1590/S167877572005000200006

Ribeiro, M.A.G., R.L.C. Albuquerque-Junior, L.M.P. Ramalho, A.L.B. Pinheiro and L.R. Bonjardim et al., 2009b. Immunohistochemical assessment of myofibroblasts and lymphoid cells during wound healin in rats subjected. Photomed. Laser Surgery, 27: 49-55. DOI: 10.1089/pho.2007.2215

Ribeiro, MA.G., R.L.C. Albuquerque-Junior, A.L.S. Barreto, V.G.M. Oliveira and T.B. Santos et al., 2009a. Morphological analysis of second-intention wound healing in rats submitted to $16 \mathrm{~J} / \mathrm{cm} 2 \lambda 660$-nm laser irradiation. Indian J. Dental Res., 20: 390-390.

Rodrigues, S.S.M.R.G., B.S.S. Maior, D.R. Aquino and A.L. Anbinder, 2009. Effects of low power laser under different protocols, in the repair of skin wounds in rats. Clin. Pesquisa Odontol. Unitau, 1: 31-37.
Sassioto, M.C.P., C.M. Inouye, R.D. Aydos, A.S. Figueiredo and E.R.J.C. Pontes et al., 2004. Study of bone repair with bovine bone devitalized matrix and calcitonin in rats. Acta Cirurgica Brasileira, 19: 495503. DOI: 10.1590/S0102-86502004000500007

Silva, E.M., S.P. Gomes, L.M. Ulbrich and A.F. Giovanini. 2007. Histological evaluation of the effects of LLLT on the epithelial, connective and osseous tissue healing: Experimental study in rats. Revista Sul-Brasileira Odontologia, 4: 29-35.

Silva, F.B., J.N. Almeida and S.M.G. Souza, 2004. Natural medicaments in endodontics -- a comparative study of the anti-inflammatory action. Brazilian Oral Res., 18: 174-179. PMID: 15311323

Torneck, C.D., 1966. Reaction of rat connective tissue to polyethylene tube implants. Oral Surgery Oral Med. Oral Pathol., 21: 379-387. PMID: 5216747

Valera, M.C., M.R. Leonardo, A. Consolaro and F.S. Matuda, 2004. Biological compatibility of some types of endodontic calcium hydroxide and glass ionomer cements. J. Applied Oral Sci., 12: 294-300. DOI: $10.1590 / \mathrm{S} 1678-77572004000400008$

Verhaegen, P.D.H.M., P.P.M. Van Zuijlen, N.M. Pennings, J.V. Marle and F.B. Niessen et al., 2009. Differences in collagen architecture between keloid, hypertrophic scar, normotrophic scar and normal skin: An objective histopathological analysis. Wound Repair Regenerat., 17: 649-656. DOI: 10.1111/j.1524-475X.2009.00533.X

Zmener, O., M.B. Guglielmotti and R.L. Cabrini, 1988. Biocompatibility of two calcium hydroxide-based endodontic sealers: A quantitative study in the subcutaneous connective tissue of the rat. J. Endodontics, 14: 229-235. DOI: 10.1016/S00992399(88)80175-4 